\title{
Estructuras elásticas
}

\author{
Elastic structures
}

$<$ RESUMEN>

La idea de un entorno en el cual la estructura sea capaz de albergar y promover la ocupación del espacio por parte de ciertas comunidades de artistas locales en Berlín es el comienzo de este proyecto. Esta infraestructura propone formas de ocupación real y desplazamientos que no necesariamente corresponden a una delimitación por superficies. Por el contrario, la estructura (líneas) se disponen de manera de promover ciertos grados y escalas de uso, abriendo las posibilidades de generación artística desde la «institución patrocinadora» a una alternativa más espontánea. Este espacio de relativa ambigüedad se concibe como un campo de eventos simultáneos, y su diseño precede al de la estructura final visible. En otras palabras, es la relación y gradientes programáticas la que se activa por medio de la simulación termodinámica. Este umapa» condiciona finalmente el ensamble de estas piezas divergentes y convergentes, expandiendo, contrayendo, torciendo y doblando debido a reacciones locales

$<$ ABSTRACT >

The starting point for the design of this environment is a structure able to house and to promote the occupation of the space by some determined artistic communities from Berlin. This infrastructure suggests forms of actual occupation and flows that not necessarily relates to a definition by surfaces. By opposition the linear structures are disposed in a way they can promote certain degrees of use and scale. This allows shifting the notion of artistic creation from the usponsoring institution» to a rather more spontaneous alternative. This relatively ambiguous space is thought as a series of simultaneous events and its design precedes its visible structure. In other words, what is activated through the means of thermodynamic simulations is the programmatic gradients. This «map»ultimately conditions and defines the assembly of these divergent and converging pieces by expanding and contracting, twisting and bending themselves due their local reactions

<PALABRAS CLAVE>

ESTRUCTURA / CAMPO / EXPANSIÓN / FLUJO / OCUPACIÓN / VARIACIÓN

<KEY WORDS

STRUCTURE / FIELD / EXPANSION / FLOW / OCCUPATION / VARIATION
Este proyecto pretende establecer un vínculo entre el diseño de una estructura física arquitectónica y ciertas normas de comportamiento en un sistema. Aunque estas relaciones están lejos de ser obvias, comparten una cantidad de propiedades y expresiones propias. Por lo tanto, el análisis y la simulación de un sistema termodinámico puede ofrecer una perspectiva renovada sobre la expresión de la estructura, relacionando ambos a través de un pensamiento o análisis común. Varias consideraciones sugieren por un lado una conexión intelectual entre ambos sistemas y por otro lado, estrategias de diseño.

Bajo la tradición de la arquitectura moderna podemos identificar fácilmente dos escuelas diferentes basadas en dos formas de expresión estructural. La escuela le corbousiana asociada comúnmente con una estructura/estética de hormigón de monolítica continuidad de transmisión de cargas. Por otro lado, la escuela miesiana presenta retículas de acero con un repertorio más extenso en el proceso de construcción de su propia herramienta abstracta, la grilla. Pero al mismo tiempo, también puede decirse que esta apertura y «libertad» alcanzada a través de estos elementos neutrales realmente no provoca la aparición de diferencias internas. Es de algún modo, un tipo de campo estático que contiene todo el potencial para ser activado. Sin embargo, la estructura también ha sido ocupada como instrumento para producir diferencias internas y cambios (Cedric Prize, Constant). La intención del proyecto es 

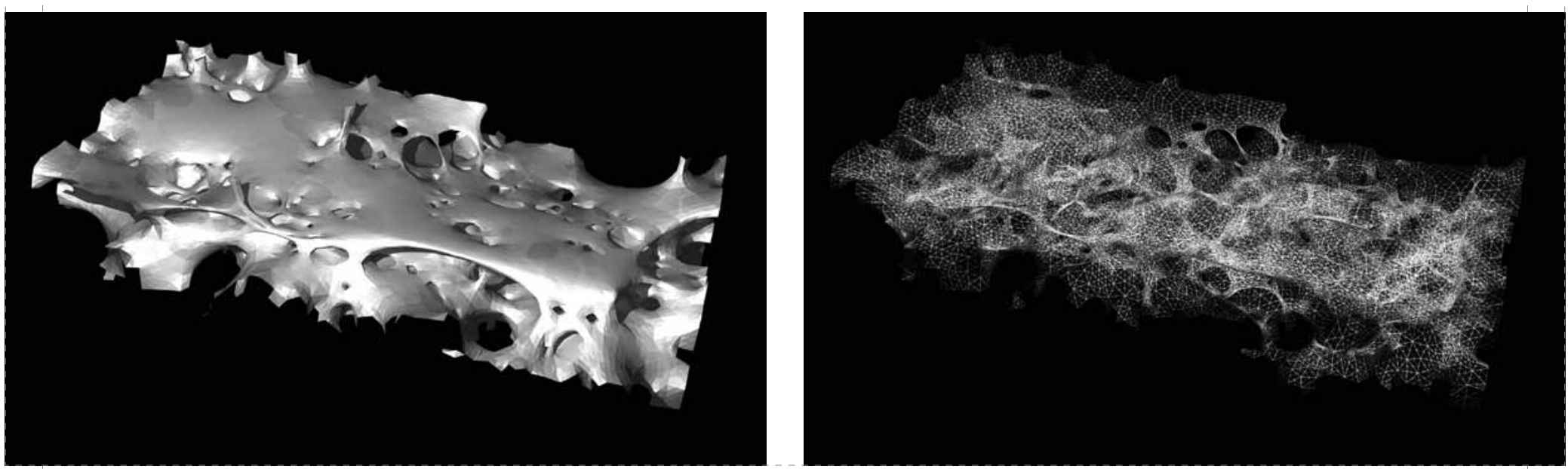

explorar la oportunidad que la estructura tiene para producir en su interior la mayor cantidad de diferencia y fricción. Esto para lograr el diferencial necesario para comenzar un set de relaciones e intercambios más complejos en su interior.

\section{Devenir de una estructura}

La materia no es pasiva o inerte. Esta activamente cambiando de acuerdo a los procesos que operan en su interior y es este permanente intercambio de energía entre cuerpos lo que los localiza dentro de un sistema. Es decir, ningún cuerpo o forma puede ser considerada separadamente de sus relaciones con los demás. Este entorno de flujo, de energía, los concatena y esa diferencia de densidades inmanente gatilla un número infinito de procesos y comportamientos en un medio determinado. Aun cuando esas energías poseen su propia «inteligencia» (como en la naturaleza autoorganizativa de temperatura, volumen y movimiento en termodinámica), no son siempre predecibles o están en estado de equilibrio, sino que en permanente autoorganización. Éstas se encuentran en permanente movimiento dentro de estos cuerpos activos definidos por flujos e intercambios, y no por umbrales o límites claros. Ésta es la naturaleza de la estructura también, cargada con energía física y capaz de alojar diferencias y deseos sociales en su interior. El desplazamiento desde una estructura neutral o en equilibrio a una estructura de «fría/caliente» más expresamente activa puede provocar que el contenido social emerja desde sus propias diferencias internas. En algún punto entre fricción y fluidez.

Por otro lado, la consistencia no puede ser considerada como un atributo interno fijo en ningún cuerpo arquitectónico, político o social. Esta es una condición que de manera análoga a la materia puede variar de sólido a fluido dependiendo al mismo tiempo de la cambiante cohesión entre sus partes y por otro lado de sus cambiantes condiciones ambientales y de las energías y fuerzas que lo cruzan. Esta cohesión se basa en campos poblados de flujos de intensidades y no en estratificaciones estables en el tiempo. El molde inicial de la

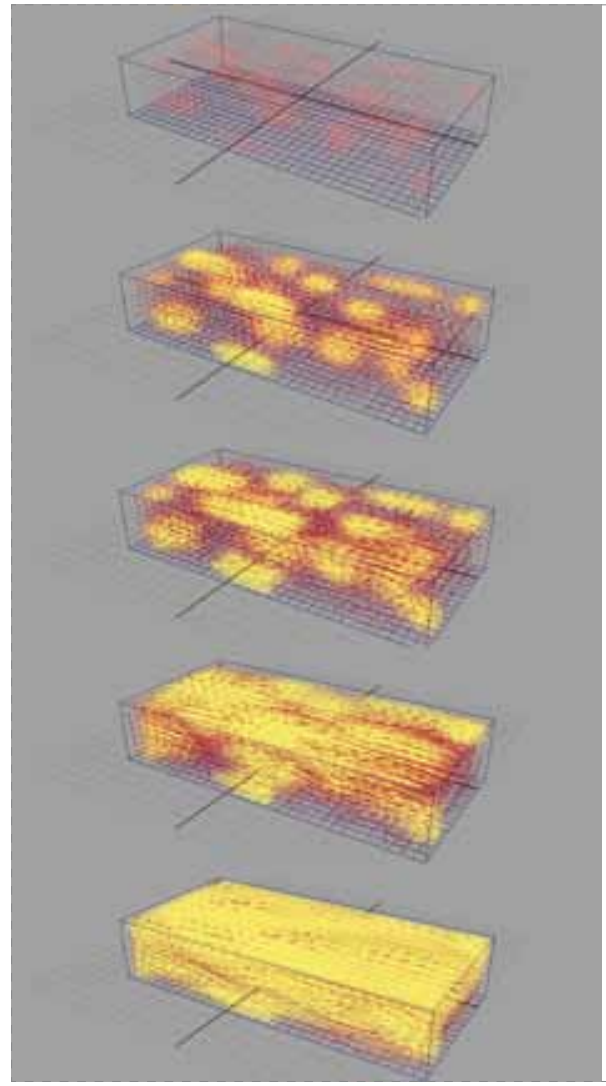

arquitectura es su propia estructura, que no puede ya ser referida como un armazón inerte sino como un cuerpo dinámico lleno de fuerzas de naturaleza física y social.

El cuerpo resultante puede ser descrito básicamente como un sistema dinámico con su propio programa de construcción, sus propias reglas de comportamiento y su propia consistencia variable e inteligente.

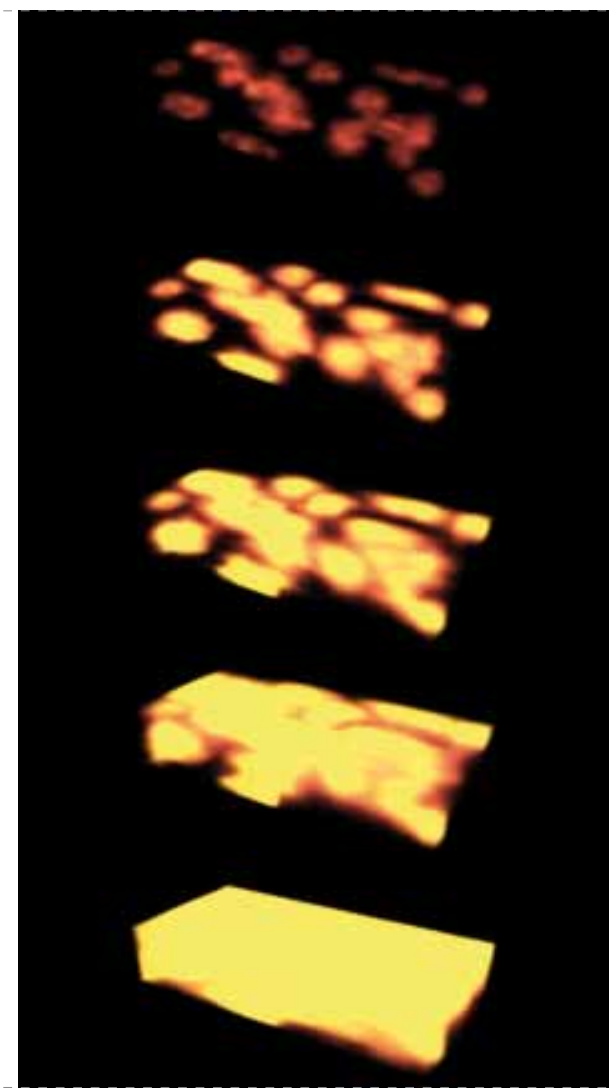




\section{Palast der Republik I Fun Pallace}

El proyecto comienza aquí con la ubicación de una serie de unidades programáticas mayores relacionadas con la producción de arte en el solar del antiguo Congreso Alemán (Palast der Republik), en Berlín, Alemania. E encargo sugiere desde el principio el diseño de una estructura para ser «tomada» o espontáneamente ocupada por artistas locales, como ha sido la tradición desde que el edificio fue desocupado durante mediados de los setenta. El programa es extraído de una serie de experiencias urbanas sin una definición formal, pero con una presencia evidente en el Berlín contemporáneo. Básicamente, no se considera una necesidad por una carga programática específica, sino un número de eventos que puedan ocurrir. La estructura alojará lo que necesita suceder en su interior

Para promover estas cargas diferenciales se necesita una estructura inicial, una que pueda asumir repetición y variación en su composición con un set de reglas para el diseño de la nueva grilla. La deriva entre estas diferencias es el objetivo mayor del proyecto. De alguna forma, emulando New Babylon de Constant ${ }^{1}$, la deriva es vista como una fuerza conducente a desplazar a los usuarios desde una rutina productiva al placer del «destruir rutas para construir y encontrar otras nuevas». Duda y búsqueda como la experiencia primaria.

\section{Armado}

En física, estas reglas, comportamientos y autoorganizaciones han sido usados para señalar cómo los fluidos y los fenómenos atmosféricos pueden ser registrados, predichos y manipulados. Por lo tanto, estas reglas pueden proveer el movimiento inicial requerido para poner en marcha otros procesos. La termodinámica entrega el análisis usado para entender y planear la transmisión de energía. Análogamente, esta información puede ser extremadamente útil en la construcción de un cuerpo social, político o arquitectónico desorganizado o desestratificado. Esto no significa que la estructura se comportará de hecho como un fluido, sino que su diseño puede heredar la inteligencia de organización (o desorganización) que tanto fluidos como gases poseen para distribuir sus diferencias internas.

Lambert, P.J.C. New Babylon. Constant. Art et Utopie. Textes Situationnistes. Cercle D'Art, 1997.

\section{Simulación}

En termodinámica, el sistema está compuesto por partículas, cuyo promedio de movimientos define las propiedades del sistema, que a su vez relaciona unas con otras a través de ecuaciones de estado o su equilibrio incipiente o provisorio. Estas propiedades pueden ser combinadas para expresar la energía interna y para determinar las condiciones para un equilibrio así como para procesos espontáneos o comportamientos emergentes. De manera similar, el sistema termodinámico puede ser considerado transmisión de energía como resultado de una fuerza causando un movimiento generalizado, y en nuestro caso, la disipación del programa y esfuerzos estructurales a través del edificio.

La representación de este sistema se realiza a través de gradientes de intensidades inscritas en un campo determinado. Principalmente, esas gradientes son la representación de una proporción de cambio al interior del campo, en el cual cada punto en el sistema se relaciona con los puntos a su alrededor inmediato de manera omnidireccional. El campo es en otras palabras, un contenedor con asignación de cualidades físicas para cada punto del espacio. El cuerpo programático/estructural se vuelve material flexible y elástico. El campo se entiende de este modo como algo expansible a través de una gran porción de espacio y por lo tanto su influencia es omnipresente.

Estos campos de vectores llenos de información dinámica se pueden obtener con animación asistida por computador a través de varios software de simulación para ingeniería y física, tal como Real Flow, Maya y otros. La simulación comienza en su estado inicial como un sistema cerrado representado por un campo de puntos estáticos en un contenedor tridimensional. Éste es una grilla simple, que no contiene aún gradientes o información dinámica. La siguiente condición del estado inicial es la localización y cualificación de los emisores de calor (positivo) o frío (negativo) cuyos tamaños, posiciones y jerarquías corresponden tanto a condiciones contextuales como programáticas. Es crucial determinar estos atributos con precisión. Como el cambio de volumen (expansión y contracción) es una variable a ser considerada, el tamaño de las unidades programáticas es reducido o aumentado en el $20 \%$. Más tarde, la simulación finalizará en cuanto la ganancia/ pérdida de volumen se complete como resultado del proceso. Se debe ser preciso en determinar las velocidades de combustión, la proporción de liberación de combustible, la cobertura y la difusión en el tiempo, entre otras muchas condiciones previas.
Es a través de la acción de estos agentes (emisores de calor/frío) que dispuestos con precisión en el espacio, que la reacción de todo el sistema comenzará. Aunque estos agentes no tienen presencia física inflectarán las condiciones del cuerpo al desplazar, expandir o comprimir masas de partículas en distintos grados o gradientes. En otras palabras, las partículas que sirven como representación de lo que sucede en la simulación no son el sistema en sí, sino que la representación entre agentes y su medio. Así, todos los parámetros y distancias entre ellos constituyen el set de relaciones elásticas, que en sí, no requiere ni de partículas o ningún otro objeto para comportarse. Posteriormente, la simulación es realizada con la grilla estática, y concluye cuando el sistema alcanza su equilibrio con un set reorganizado de puntos nuevamente estático. Acá podemos distinguir tres categorías del cuerpo elástico-térmico: desde la grilla estática al equilibrio de fluido y el inequilibrio de fluido entre ellos. Es esta categoría que, una vez registrada, se convierte en útil para el diseño de la estructura como un estado intermedio que revela esta disposición de diferencias en progreso.

\section{Diagrama}

El estado de la simulación es representado por un campo de vectores que muestran diferentes tendencias y magnitudes en cada punto del espacio. Es decir, que incluso siendo la información de cada punto de naturaleza extensiva (ejemplo: longitud, posición) la relación y representación general es de naturaleza intensiva (ejemplo: convección, aceleración). Por lo tanto, el diagrama final muestra comportamientos emergentes que no son el mero resultado de la suma o aritmética de las cualidades extensivas sino que muestra la distribución intensiva o la inteligencia de la energía corriendo a través de este sistemacuerpo, comprimiendo y expandiendo todo en su interior.

\section{Consolidación}

El resultado de la simulación, que es un momento dentro del proceso dinámico, se registra en una suerte de imagen instantánea de una estructura en movimiento. Este diagrama provee la planificación para una posterior construcción. La repetición y variación inicial de los miembros estructurales, se convierte ahora en un repertorio de sets completos de gradientes estructurales. Este se convierte a su vez en el lenguaje en el cual el contenido extraído del diagrama abstracto, será «escrito». Este se desplaza desde ser 

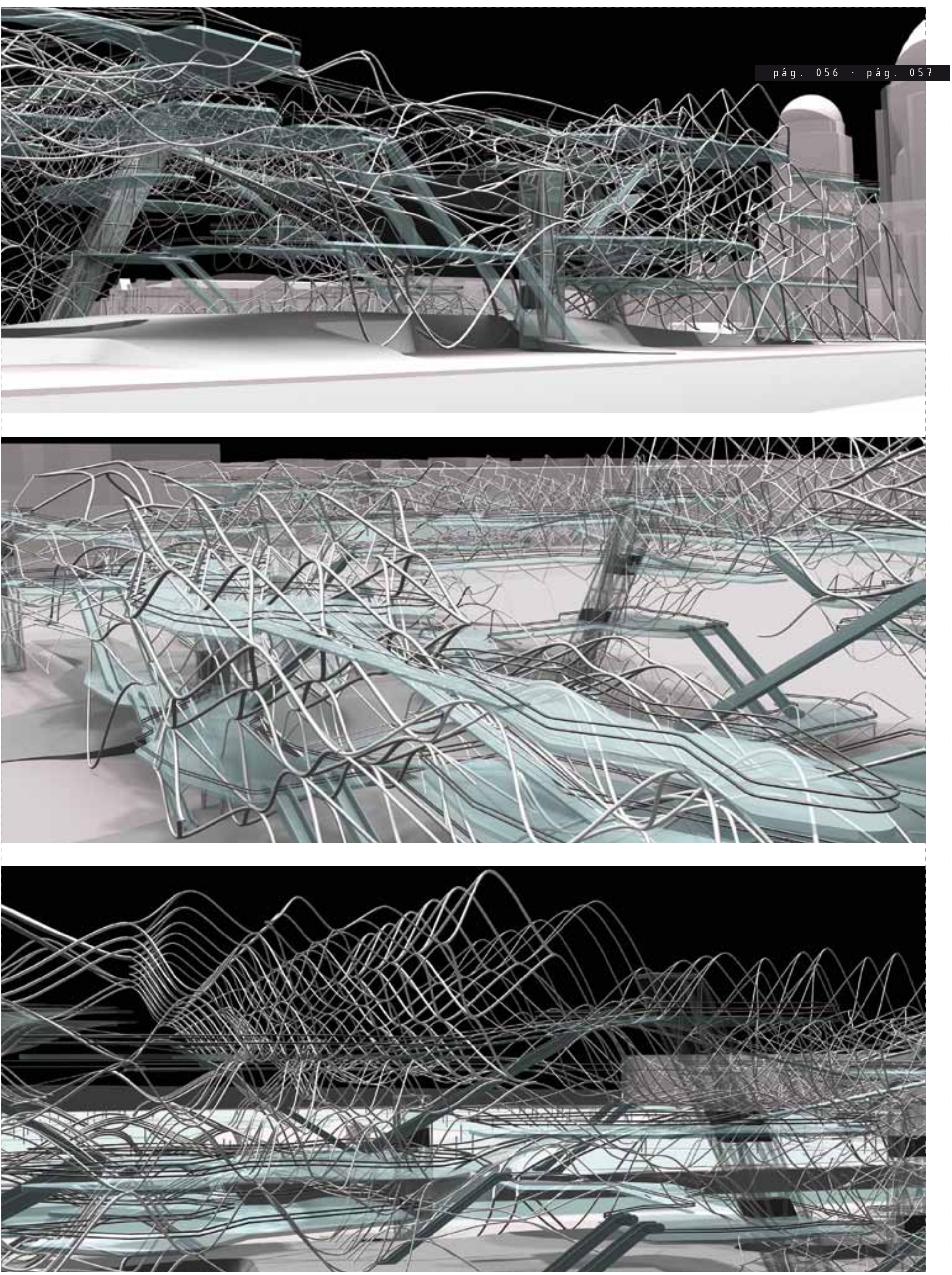
«palabras» aisladas hacia ser frases tectónicas completas.

Para conseguir una estructura capaz de contener el potencial diferencial, se debe utilizar la información entre dos estados de la simulación termodinámica (no-equilibrio) como fuente de un comportamiento en curso. Sin embargo, esta información que proviene de un campo de vectores resultante no debe ser literalmente expresada como forma, sino que como un mapa tridimensional que permite distribuir y cualificar los miembros estructurales en una configuración final. Este mapa entrega un número de magnitudes extensivas acerca de un cuerpo compuesto por magnitudes intensivas. La estructura no se comporta literalmente como un fluido, sino que es posible traducir esas cualidades térmicas a las cualidades materiales de los miembros estructurales tales como: dimensiones, curvatura, tensión y torsión entre muchas otras, a cada punto de la estructura. El resultado es un cuerpo-estructura que se muestra a sí mismo como una desorganización en proceso. Ni sólido ni fluido sino elástico. Su composición se hace a partir de miembros que se expanden, colapsan, comprimen, tuercen, curvan y densifican en distintas gradientes de acuerdo a la disposición general en movimiento. El potencial contenido en esta estructura yace en su habilidad para albergar y distribuir diferencias; la diferencia en sí misma actúa como el combustible para generar el emerger más espontáneo de deseos y aspiraciones sociales en su interior.

\section{Reflexión final}

La supremacía de la superficie como analogía computacional básicamente gobernó buena parte de los discursos arquitectónicos durante los últimos 10 años. Sin embargo, es la hipersimplificación de la hipersuperficie lo
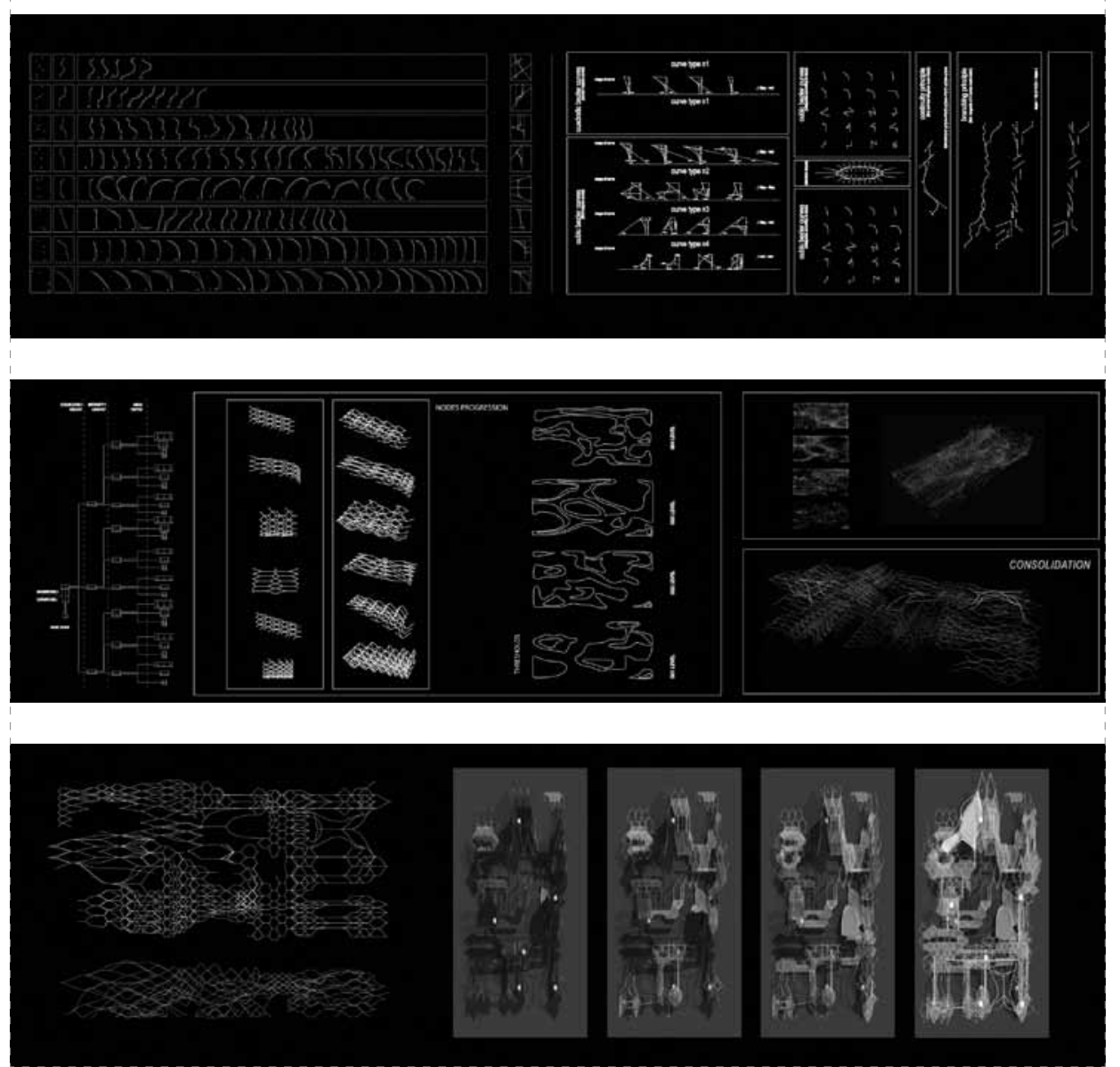

que finalmente erradica el potencial de la diferencia y la fricción como principio social. La traducción directa de «diferencias» en tanto son aspiraciones sociales, quedan reducidas a la continuidad de una superficie inerte producida en el computador. La hipercualificación de estas superficies puede eventualmente activar ciertos comportamientos más bien libres de carga programática, a través de la codificación de estos lugares. Sin embargo, es la expresión de estas diferencias, a través de la materia y las energías que fluyen, lo que finalmente puede generar el mayor potencial de fricción, sugestión y diferencia necesaria para gatillar un conflicto versus la negociación de estos espacios. 